\title{
Saúde do trabalhador em tempos de COVID-19: a experiência do município de Londrina
}

\author{
Michelle Moreira Abujamra Fillis, Mara Solange Gomes Dellaroza, Rosilene Aparecida \\ Machado, Tatiane Aparecida Alves Pelaquim, Valdelice Vaz Coelho, Valéria Cristina Almeida \\ de Azevedo Barbosa, Vânia Cristina da Silva Alcantara, Celita Salmaso Trelha
}

\section{RESUMO}

A pandemia da COVID-19 está sendo um grande desafio para a saúde e o processo de trabalho como um todo deve ser considerado na elaboração e implantação de estratégias para seu enfrentamento. A necessidade de distanciamento social para evitar a proliferação rápida do vírus, de acordo com as orientações da Organização Mundial de Saúde, levou milhares de pessoas ao trabalho remoto, fechamento de indústrias, serviços e escolas em todos os níveis de ensino. Diante do exposto, o objetivo do presente estudo é relatar a experiência de um programa de prevenção à saúde do trabalhador no enfrentamento da pandemia do COVID-19 no município de Londrina e região.

Palavras-chave: Infecções por Coronavirus; Saúde do Trabalhador; Atenção Primária à Saúde; Educação em Saúde; Comunicação por Videoconferência.

\section{ABSTRACT}

The COVID-19 pandemic is a major health challenge and the work process as a whole must be considered in the elaboration and implementation of strategies to face it. The need for social distance to avoid the rapid proliferation of the virus, according to the guidelines of the World Health Organization, has led thousands of people to remote work, closing industries, services and schools at all levels of education. Given the above, the objective of the present study is to report the experience of a program of prevention in workers' health in coping with the pandemic of COVID-19 in the city of Londrina and region.

Keywords: Coronavirus Infections; Occupational Health; Primary Health Care; Health Education; Videoconferencing Communication.
Revista da Rede APS 2020

Publicada em: 09/06/2020

DOI:10.14295/aps.v2i2.97

Michelle Moreira A. Fillis (Universidade Estadual de Londrina e Secretaria Municipal de Saúde de Londrina, Londrina, PR, Brasil)

Mara Solange G. Dellaroza Celita Salmaso Trelha (Universidade Estadual de Londrina, Londrina, PR, Brasil)

Rosilene Aparecida Machado Tatiane Aparecida A. Pelaquim Valdelice Vaz Coelho Valéria Cristina A. A. Barbosa Vânia Cristina S. Alcantara (Secretaria Municipal de Saúde de Londrina, Londrina, PR, Brasil)

Correspondência para: Michelle Moreira A. Fillis michellemoreira@uel.br

Mara Solange G. Dellaroza dellaroza@uel.br

Rosilene Aparecida Machado rosilenemachado@gmail.com 


\section{INTRODUÇÃO}

A COVID-19 está sendo um grande desafio para a saúde e o processo de trabalho deve ser considerado na elaboração e implantação de estratégias de enfrentamento a essa pandemia. Baker, Peckham, Seixas ${ }^{1}$ descreveram que tanto o exercício das atividades laborais quanto as condições de trabalho são fontes potenciais de exposição ao vírus e território de disseminação da doença.

A literatura mostra que casos de contaminação foram atribuídos ao exercício profissional de diversas categorias de trabalho. Há uma longa lista de trabalhos que envolvem contato direto com o público e proximidade física, como trabalhadores do comércio, prestadores de serviços, com destaque aos entregadores que correm um risco maior de exposição devido ao grande número de contatos diários ${ }^{2}$.

O provável foco inicial de contaminação pelo manuseio de animais vivos, na cidade de Wuhan na China, foi de trabalhadores e clientes de um mercado atacadista de frutos do $\mathrm{mar}^{3}$. Em Singapura, 68\% dos 25 casos iniciais de contaminação comunitária foram atribuídos ao exercício profissional ${ }^{4}$. No Brasil, o segundo óbito por coronavírus registrado foi o caso de uma empregada doméstica do Rio de Janeiro ${ }^{5}$. Além disso, há inúmeros casos de profissionais de saúde acometidos pela doença diretamente envolvidos com os cuidados aos pacientes contaminados. Os profissionais de saúde apresentam risco maior de exposição a alta carga viral, devido ao contato próximo com pacientes com COVID-19, o que os coloca em risco de serem infectados. Ao mesmo tempo, eles próprios são uma fonte importante de transmissão da doença para colegas, pacientes, amigos e familiares. Na Itália, foi relatado que mais de 5.000 profissionais de saúde haviam testado positivo para o Coronavírus e mais de 40 haviam morrido como resultado da COVID-196 .

Toda atividade laboral e todo trabalhador precisa ser considerado e preparado, não apenas para a sua proteção, mas também para compreender que sua atividade tem um papel importante no combate à pandemia ${ }^{7}$. A preservação da saúde de todos os profissionais que se mantêm trabalhando, é fundamental para controlar a disseminação da doença e para a manutenção das pessoas em isolamento, confinamento ou quarentena. Dessa forma, medidas de prevenção são necessárias para os trabalhadores em atividades com maior risco de exposição.

Não é trivial preparar um robusto sistema na atenção primária em saúde (APS) para responder com qualidade a situações de emergência pública. Há inúmeras evidências que demonstram influência significativa na redução de mortalidade e desigualdades em saúde, o que tende a ser potencializado pela combinação com políticas de transferência de renda e proteção social ${ }^{8}$. No Brasil, com seu Sistema Único de Saúde, com a Estratégia de Saúde da Família (ESF) e com o trabalho do Núcleo Ampliado de Saúde da Família (NASF), a prática da Atenção Primária à Saúde é absolutamente central para a resposta local ao COVID-19.

De modo a romper com a visão fragmentada tradicional da atenção à saúde no Brasil, os NASFs atuam de forma integrada, prestando apoio matricial às equipes de Saúde da Família, capacitando-as para intervenções terapêuticas e pedagógicas entre populações específicas, como, por exemplo, nos consultórios de rua, na assistência a populações ribeirinhas e fluviais, ou no Programa Academia da Saúde. O trabalho do NASF contribui para o acesso da população à equipe multiprofissional da ESF, de forma a direcionar as ações da Atenção Básica (AB) para o desenvolvimento de estratégias de promoção, prevenção e reabilitação da saúde da população ${ }^{9}$.

A partir disso, o Ministério da Saúde regulamentou o uso de telemedicina, excepcionalmente para o contexto da epidemia de COVID-19, com fins de prestação de abordagem pré-clínica, suporte assistencial, consulta, monitoramento e diagnóstico em todo 
o sistema de saúde brasileiro. Este foi um passo fundamental para que o teleatendimento passasse a constar oficialmente no protocolo nacional de manejo clínico deste problema no contexto da APS, o que resultou, entre outras coisas, na adoção por estados e municípios de centrais de teleatendimento como componentes essenciais de primeiro contato das pessoas com a rede de atenção à saúde ${ }^{10}$.

Diante do exposto, o objetivo do presente estudo é relatar a experiência de um programa de prevenção à saúde do trabalhador no enfrentamento da pandemia da COVID-19 no município de Londrina e região.

\section{A EXPERIÊNCIA}

Londrina é um município localizado no interior do estado do Paraná, na região sul do Brasil, distanciando $381 \mathrm{~km}$ da capital. Tem uma população de 569.733 habitantes segundo a estimativa do IBGE em 201911, além da zona urbana, possui uma extensa zona rural com 13 distritos. No município, a ESF tem-se mantido como estratégia principal para a organização da APS. Essa estratégia direciona-se à população de um território adscrito, com o cuidado centrado na pessoa e favorece a reorientação dos processos de trabalho, melhorando a resolutividade dos serviços e, consequentemente, a qualidade de vida e satisfação do usuário. O município tem 54 Unidades Básicas de Saúde (UBS), destas 12 estão localizadas na região rural. São 71 equipes da ESF distribuídas em todas as unidades de saúde, e compostas por médico, enfermeiro, auxiliar de enfermagem e agente comunitário de saúde. O NASF-AB, permanece organizado com foco no território de modo a prestar apoio matricial, tanto às equipes da $E S F$, quanto às equipes da $A B$. É estruturado de modo a realizar atendimento compartilhado e interdisciplinar $\mathrm{e}$ possui dez equipes compostas pelos seguintes profissionais: farmacêutico, nutricionista, educador físico, psicólogo e fisioterapeuta e duas equipes NASF modalidade Residência em Saúde da Família em parceria com a Universidade Estadual de Londrina (UEL).

Em relação ao trabalho, no município de Londrina há 203.294 pessoas ocupadas, o que representa $36,4 \%$ da população total, e com salário médio dos trabalhadores formais de 2,8 salários mínimos segundo dados do IBGE em $2017^{11}$

Diante da pandemia da COVID-19, várias ações foram desencadeadas pela gestão local com o objetivo de reduzir o seu impacto na população e de preparar a rede de assistência para o enfrentamento da doença no município. Dentre as ações, a principal delas foi a instituição, pelo Executivo Municipal, do Comitê de Operações de Emergência em Saúde Pública para o enfrentamento da COVID-19 (COESP MunicipalCOVID-19), com a participação de representantes técnicos de todos os serviços de saúde do município, dos setores público e privado $^{12}$.

Além disso, foram organizadas seis UBS, uma em cada região da cidade, sendo duas em região com maior densidade populacional, para atendimento do indivíduo com sintomas respiratórios, uma unidade de pronto atendimento de urgência (UPA) e o Hospital Universitário da Universidade Estadual de Londrina (UEL) como referência para a internação.

Em Londrina, até o dia 28 de maio de 2020, foram 340 casos confirmados da doença, 21 óbitos e 183 se recuperaram e tiveram alta (Tabela 1). Segundo boletim informativo, destes 340 casos confirmados, $56 \%$ eram do sexo feminino, 145 (42,6\%) tinham de 20 a 39 anos, 119 (35\%) tinham de 40 a 59 anos, e $65(19,1 \%)$ tinham 60 anos ou mais ${ }^{12}$. 
Tabela 1 - Distribuição dos casos de COVID-19 em Londrina, no Paraná, Brasil e Mundo, 2020.

\begin{tabular}{lcccc}
\hline \multicolumn{1}{l}{ REGIÃO } & LONDRINA & PARANÁ & BRASIL* & MUNDO \\
\hline Confirmados & 340 & 3.984 & 411.821 & 5.929 .312 \\
Curados & 183 & 1.806 & 166.647 & 2.387 .896 \\
Óbitos & 21 & 169 & 25.598 & 357.781 \\
$\begin{array}{l}\text { Aguardando } \\
\text { Exames } \\
\text { Descartados }\end{array}$ & 337 & - & - & - \\
\hline
\end{tabular}

*Brasil dados referentes ao dia 28/05/2020

Fonte: AMSL (2020)느.

O aparecimento dos casos iniciou em 16/03/2020 e a evolução é crescente como pode ser observada na Figura 1.

O projeto intitulado "Saúde do Trabalhador em Tempos de COVID-19: como se proteger e evitar a disseminação" tem por objetivo orientar trabalhadores do município de Londrina e região sobre medidas de proteção e prevenção à COVID-19.
Foi desenvolvido em parceria entre a Secretaria Municipal de Saúde de Londrina e integrantes do Projeto de Extensão UEL pela Vida, contra o coronavírus. Participam profissionais do NASF e ESF que por integrarem grupos de risco para COVID-19 estão trabalhando de forma remota, além de professores e estudantes do Curso de Fisioterapia e Enfermagem da UEL.

Figura 1 - Casos confirmados, curados e óbitos por Covid-19. Londrina, PR, 16/03/2020 a $28 / 05 / 2020$.

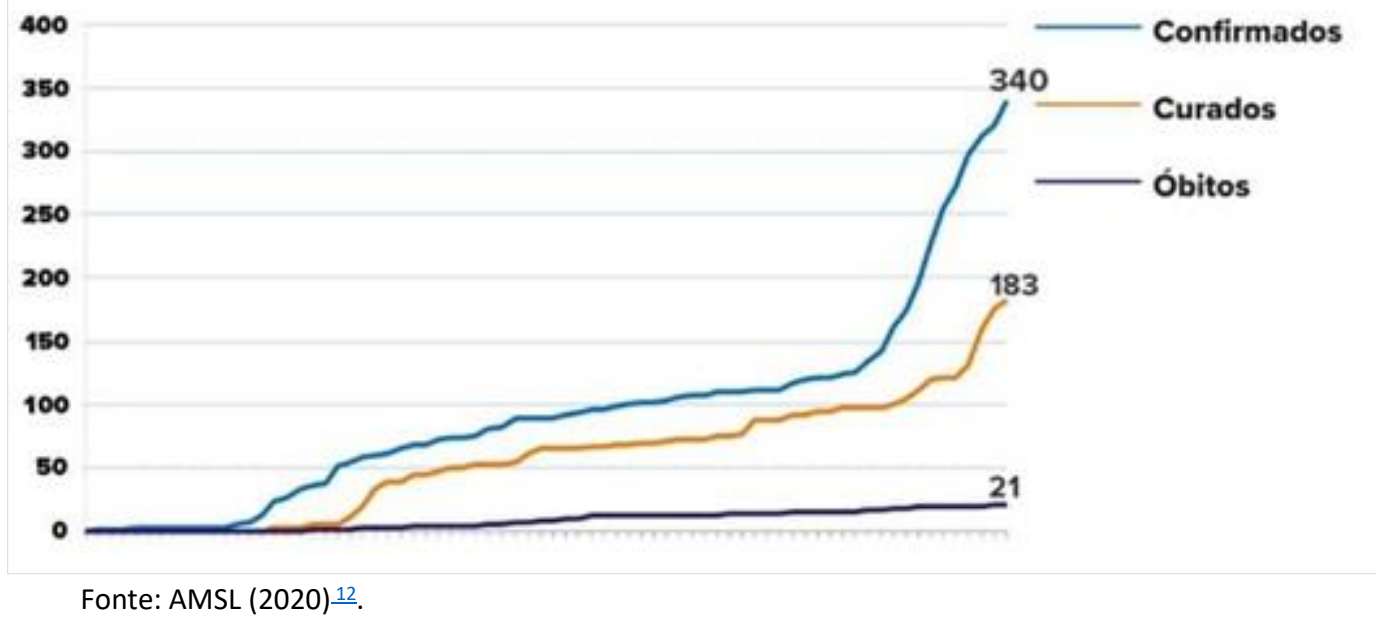

A população alvo constitui-se de trabalhadores de todas as categorias profissionais dos segmentos do comércio, indústria, construção civil, prestação de serviço, empresas e seus respectivos sindicatos, associações, escolas e condomínios comerciais e residenciais.

Inicialmente os profissionais da ESF e do NASF foram matriciados sobre ferramentas tecnológicas e uso de videoconferências para 
promover a educação em saúde. Além disso, foi realizada divulgação em mídias sociais, UBS do município de Londrina e busca ativa por contato telefônico com os setores e agendamento dos interessados.

Após o esclarecimento sobre o projeto, foi realizado agendamento e orientação do uso das ferramentas virtuais e realizado o treinamento por meio de videoconferências que duravam em média de 60 minutos. 0 treinamento consistiu em informações sobre a doença da COVID-19 (formas de transmissão, sintomas, automedicação, quando e onde procurar o serviço de saúde); como manter o distanciamento social; uso adequado de máscaras e Equipamentos de Proteção Individual; Lavagens das mãos e orientações ao tossir e espirar, de higiene com dispositivos e equipamentos no trabalho, no transporte público e ao chegar em casa; limpeza e desinfecção, entre outros. Também são fornecidas informações específicas para cada categoria profissional e local de trabalho.

Em relação ao uso de máscaras, em Londrina foi determinado o uso obrigatório em todos os locais públicos por meio do Decreto № 484 de 17 de abril de 202013 da Prefeitura Municipal de Londrina. Os trabalhadores foram instruídos quanto ao uso e tempo correto da utilização da máscara, bem como o procedimento apropriado de lavagem e como confeccioná-la.

Após o treinamento, os participantes recebem por e-mail materiais de apoio referentes ao tema abordado e fizeram uma avaliação por meio de formulário online.

\section{IMPACTOS, FACILIDADES E DIFICULDADES}

Foram orientadas 21 empresas, sendo 13 de prestação de serviço (condomínios comerciais e residenciais, salões de beleza e estética, escolas), 5 da construção civil (construtoras) e 3 indústrias, com treinamento de cerca de 109 multiplicadores atingindo cerca de 2.500 funcionários no período de 30 dias.

Por meio de formulário online, foram recebidas respostas de $56(51,4 \%)$ dos participantes do treinamento, sendo que $44(78,6 \%)$ eram do sexo feminino e 24 (42,9\%) tinham ensino superior completo. Do total de participantes $67,5 \%$ eram funcionários de escola, 16,9\% prestadores de serviço, $10,7 \%$ da construção civil, $8,9 \%$ do comércio e 3,6\% síndicos de condomínios. Quando questionados sobre sentir-se preparado para lidar com a COVID-19 no trabalho, apenas 25 (44,6\%) se consideravam aptos e 33,3\% referiram não utilizar equipamentos de proteção individual no trabalho. Além disso, 96,4\% relataram que lavavam as mãos frequentemente e todos consideravam importante o uso da máscara de tecido para proteção. Aproximadamente 95\% dos participantes consideraram o treinamento importante e todos indicariam para outra empresa.

Poucos condomínios aderiram ao treinamento, nos quais participaram síndicos e funcionários de vários setores como administrativo, portaria, zeladoria, garagem, entre outros. Verificou-se que parte deles detinham domínio parcial das informações, demonstrando-o por meio de questionamentos, por exemplo, sobre o uso e o tempo correto da máscara e o procedimento apropriado diante da existência de um morador que apresentar resultado positivo para COVID19. As dúvidas apresentadas pelos participantes foram esclarecidas durante o treinamento, tanto oralmente quanto de forma escrita (via chat de apoio) com suporte de outros servidores que integram projeto. Ressalta-se que surgiram indagações cujas informações não constavam do escopo do projeto que, no entanto, foram sanadas pela equipe de apoio que rapidamente obtiveram a informação em fonte segura. Ao aprofundar nas especificidades dos condomínios com medidas de prevenção especificas, surgiram peculiaridades que não se aplicam a outras áreas. Da mesma forma, foi ressaltada a importância dos funcionários no combate à COVID-19, seja pela sua própria 
higiene e dos ambientes, seja em propagar informações aos moradores sobre os meios de prevenção e entre os próprios colaboradores.

No mesmo sentido, os cuidados pelos condôminos são indispensáveis em relação à circulação nas áreas comuns e sua higiene, a viabilidade ou não de sua utilização, percebeuse também a importância do síndico na liderança do condomínio em relação a organização dos funcionários para receber o treinamento e implementar mudanças.

Em relação à construção civil, um extenso protocolo determinado pela prefeitura foi implementado pelas construtoras nos ambientes de trabalho, associado a orientações da Câmara Brasileira da Indústria da Construção (CBIC), que congrega as entidades regionais do setor empresarial da construção, que apresentou recomendações para adotarem no canteiro de obras, na execução dos trabalhos de construção, com base nas particularidades do setor, bem como nas orientações das autoridades públicas de saúde ${ }^{14}$.

Após realização de contato com o sindicato responsável, houve adesão voluntária de três empresas, facilitada por parcerias anteriores em ações de prevenção à saúde. Após, 30 dias, e com piora do cenário epidemiológico de Londrina, outros três construtores solicitaram a orientação, sendo uma delas responsável por obras em andamento em toda a região Sul do país. Foi realizado treinamento dos técnicos de segurança para que eles disseminassem as informações entre os trabalhadores nos canteiros com o foco na prevenção ao novo coronavírus, para evitar contágio e transmissão no local de trabalho, evitando novos casos nas famílias desses trabalhadores, com as medidas praticadas em suas casas também.

Após as orientações, perceberam-se algumas necessidades de readequação, como por exemplo, uma ocasião em que nos questionaram se, após lavar as mãos, ao invés do papel toalha, poderia ser utilizada a máquina de ar fornecida pela empresa. Esses aparelhos podem aumentar a dispersão de gotículas e facilitar a transmissão do vírus, e foi, portanto, sugerida a adaptação na empresa.

Os trabalhadores participantes demonstraram interesse sobre o assunto, participando de forma ativa com questionamento e sanando dúvidas.

\section{CONSIDERAÇõES FINAIS}

Esse projeto tem proporcionado aos trabalhadores mais conhecimento sobre a COVID-19 e as formas de prevenção da doença, possibilitando que essas orientações possam ser multiplicadas no ambiente de trabalho e levada a familiares e amigos. Além disso, contribui para fortalecer as ações dentro dos serviços de saúde, somando forças com os profissionais de saúde do município. 


\section{NOTAS E REFERÊNCIAS}

1- Baker MG, Peckham TK, Seixas NS. Estimating the burden of United States workers exposed to infection or disease: a key factor in containing risk of COVID-19 infection. Med Rxiv. 2020. Disponível em: https://doi.org/10.1101/2020.03.02.20030288.

2- Burdorf A, Porru F, Rugulies R. The COVID-19 (Coronavirus) pandemic: consequences for occupational health. Scand J Work Environ Health 2020;46(3):229-230. doi:10.5271/sjweh.3893

3- Li Q, Guan X, Wu P, Wang X, Zhou L, Tong Y, et al. Early transmission dynamics in Wuhan, China, of novel coronavirus-infected pneumonia. N Engl J Med. 2020; 382:1199-207. Disponível em: https://doi.org/10.1056/NEJMoa2001316.

4- Koh D. Occupational risks for COVID-19 infection. Occup Med. 2020;70(1):3-5. Disponível em: https://doi.org/10.1093/occmed/kqaa036.

5- BBC News Brasil [Internet]. São Paulo: Vinícius Lemos; 2020. Ministério Público do Trabalho analisa morte de doméstica no RJ após patroa ter coronavírus; [aproximadamente 2 telas]. Disponível em: https://www.bbc.com/portuguese/brasil-51982465.

6 -Giuffrida A, Tondo L. 'As if a storm hit': more than 40 Italian health workers have died since crisis began. The Guardian. 26 March 2020. https://www.theguardian.com/world/2020/mar/26/as-if-a-storm-hit-33italian-health-workers-have-died-since-crisis-began.

7- Jackson Filho JM, Ávila, AA, Garcia AE, Garcia E, Akiyoshi SC, Maeno M. A saúde do trabalhador e o enfrentamento da COVID-19. Rev. Bras. Saúde Ocup. [Internet]. 2020;45: e14. Available from: http://www.scielo.br/scielo.php?script=sci_arttext\&pid=S0303-76572020000100100\&Ing=en. Epub Apr 17, 2020. https://doi.org/10.1590/2317-6369ed0000120.

8- Sarti TDias et al . Qual o papel da Atenção Primária à Saúde diante da pandemia provocada pela COVID19?. Epidemiol. Serv. Saúde, Brasília, 2020;29(2):e2020166. Available from <http://www.scielo.br/scielo.php?script=sci_arttext\&pid=S2237-

96222020000200903\&Ing=en\&nrm=iso>. access on 30 May 2020. Epub Apr 27, 2020. https://doi.org/10.5123/s1679-49742020000200024.

9- Seus TLC et al. Núcleo de Apoio à Saúde da Família: promoção da saúde, atividade física e doenças crônicas no Brasil - inquérito nacional PMAQ 2013. Epidemiol. Serv. Saúde, Brasília, 2019; 28(2): e2018308. Available from http://www.scielo.br/scielo.php?script=sci_arttext\&pid=S223796222019000200307\&Ing=en\&nrm=iso>. access on 30 May 2020. Epub June 27, 2019. https://doi.org/10.5123/s1679-49742019000200009.

10- Brasil. Ministério da Saúde. Portaria MS/GM no 467, de 20 de março de 2020. Dispõe, em caráter excepcional e temporário, sobre as ações de Telemedicina, com o objetivo de regulamentar e operacionalizar as medidas de enfrentamento da emergência de saúde pública de importância internacional previstas no art. 3으 da Lei no 13.979, de 6 de fevereiro de 2020, decorrente da epidemia de COVID-19 [Internet]. Diário Oficial da União, Brasília (DF), 2020 mai 29. Disponível em: http://www.in.gov. br/en/web/dou/-/portaria-n-467-de-20-de-marcode-2020-249312996

11- Instituto Brasileiro de Geografia e Estatística. Rio de Janeiro: IBGE; 2020 [acessado em 30 mai. 2020]. Disponível em:<URL: https://cidades.ibge.gov.br/brasil/pr/londrina/panorama 
12- Autarquia Municipal de Saúde de Londrina. III Informe epidemiológico. https://saude.londrina.pr.gov.br/index.php/dados-epidemiologicos/informe-epidemiologico.html

13- Prefeitura de Londrina. Decreto № 484 de 17/04/2020: Estabelece condições para retomada das atividades comerciais e de prestação de serviços no Município de Londrina e dá outras providências.

14- Câmara Brasileira da Indústria da Construção (CBIC). Disponível em: https://cbic.org.br/wpcontent/uploads/2020/03/A_pandemia_do_coronavirus_v2.pdf. 